\title{
La tente et le campement chez les Touareg Kel Ferwan
}

M. Dominique Casajus

Citer ce document / Cite this document :

Casajus Dominique. La tente et le campement chez les Touareg Kel Ferwan. In: Revue de I'Occident musulman et de la Méditerranée, n³2, 1981. pp. 53-70;

doi : https://doi.org/10.3406/remmm.1981.1919

https://www.persee.fr/doc/remmm_0035-1474_1981_num_32_1_1919

Fichier pdf généré le 21/04/2018 


\title{
Résumé
}

Nous livrons ici une série de faits ethnographiques recueillis chez les Touaregs Kel Ferwan (nord du Niger) et restes jusqu'ici un peu inaperçus. La tente des Kel Ferwan est considérée comme le domaine de la femme. Tout d'abord la tente où vit un couple appartient à l'épouse. Les tentes se transmettent de mère en fille. Ensuite, une femme tend à être identifiée à sa tente. Par ailleurs, la tente est considérée comme une représentation du cosmos et elle est construite selon un modèle immuable depuis, dit-on, le debut des temps. Les tentes sont regroupées en campements, qui constituent des unités de résidence patrilocale. Le campement est le domaine des hommes. Au niveau du campement, la société s'inscrit dans l'histoire et la temporalité. La tente et le campement s'opposent donc comme domaine des femmes et domaine des hommes et aussi comme lieu de l'immuable et du temporel.

\begin{abstract}
Here are a series of ethnographical facts collected from the Kel Ferwan Touaregs (in the north of Niger) and which up to now have been granted little attention. For the Kel Ferwan the tent is considered as pertaining to the women's domain. First of all the tent where a couple lives belongs to the wife. Moreover it is handed down from mother to daughter, and then a woman tends to be identified with her tent. The tent is considered as symbolizing the cosmos, and it is said to be built according to a pattern unaltered from beginning of times. The tents are gathered to form encampments, which constitute patrilocal units. The encampment is the domain of the men, the place where society pertains to history and time. So that the tent and the encampement are opposite, one being the domain of the women and of immutability, the other that of the men and of history.
\end{abstract}




\title{
LA TENTE ET LE CAMPEMENT CHEZ LES TOUAREgS KEL FERWAN (1)
}

\author{
par Dominique CASAJUS
}

\begin{abstract}
Nous nous proposons ici d'évoquer brièvement la façon dont les Touaregs Kel Ferwan mettent en œuvre leur nomadisme, et de quelles valeurs sont chargés les moyens de cette mise en œuvre. Cet article est donc d'abord la présentation de quelques données ethnographiques, dont quelques-unes touchent à des domaines de la vie touarègue un peu négligés jusqu'ici; cette présentation est accompagnée de quelques indications touchant à l'idéologie, aux "représentations ". Sur ce dernier point, que nous comptons développer dans des publications ultérieures, nous resterons bref; mais nous croyons là aussi que ces indications sommaires peuvent constituer une introduction à une compréhension un peu renouvelée de l'idéologie touarègue.

On sait que les Kel Ferwan, auxquels J. Nicolaisen a consacré une partie de son livre Culture and ecology of pastoral Twaregs (Copenhague 1963) nomadisent à l'ouest et au sud d'Agadez; ils forment un ensemble de tribus reconnaissant une certaine prééminence à l'une d'entre elles, celle des Irawatan, dite " noble " (2). Nous ferons ça et là des remarques comparatives à propos d'autres Touaregs, en particulier les Kel Ewey qui vivent au nord des terres Kel Ferwan, essentiellement dans le massif de l'Aïr.
\end{abstract}

\section{Translittération}

Le r grasseyé est transcrit $g h$, la chuintante sh et le ou w.

é correspond au phonème que K.G. Prasse note par un e renversé. Il représente la voyelle zéro et a une valeur plus phonologique que phonétique. Sa valeur est le plus souvent proche du e muet français. Le $e$ correspond ici au $e ́$ français.

$\ddot{a}$ correspond à un timbre intermédiaire entre le $a$ et le $e$, d'une valeur voisine du $\dot{e}$ français. Le $\dot{a}$ et le $\dot{e}$ désignent les voyelles longues. 


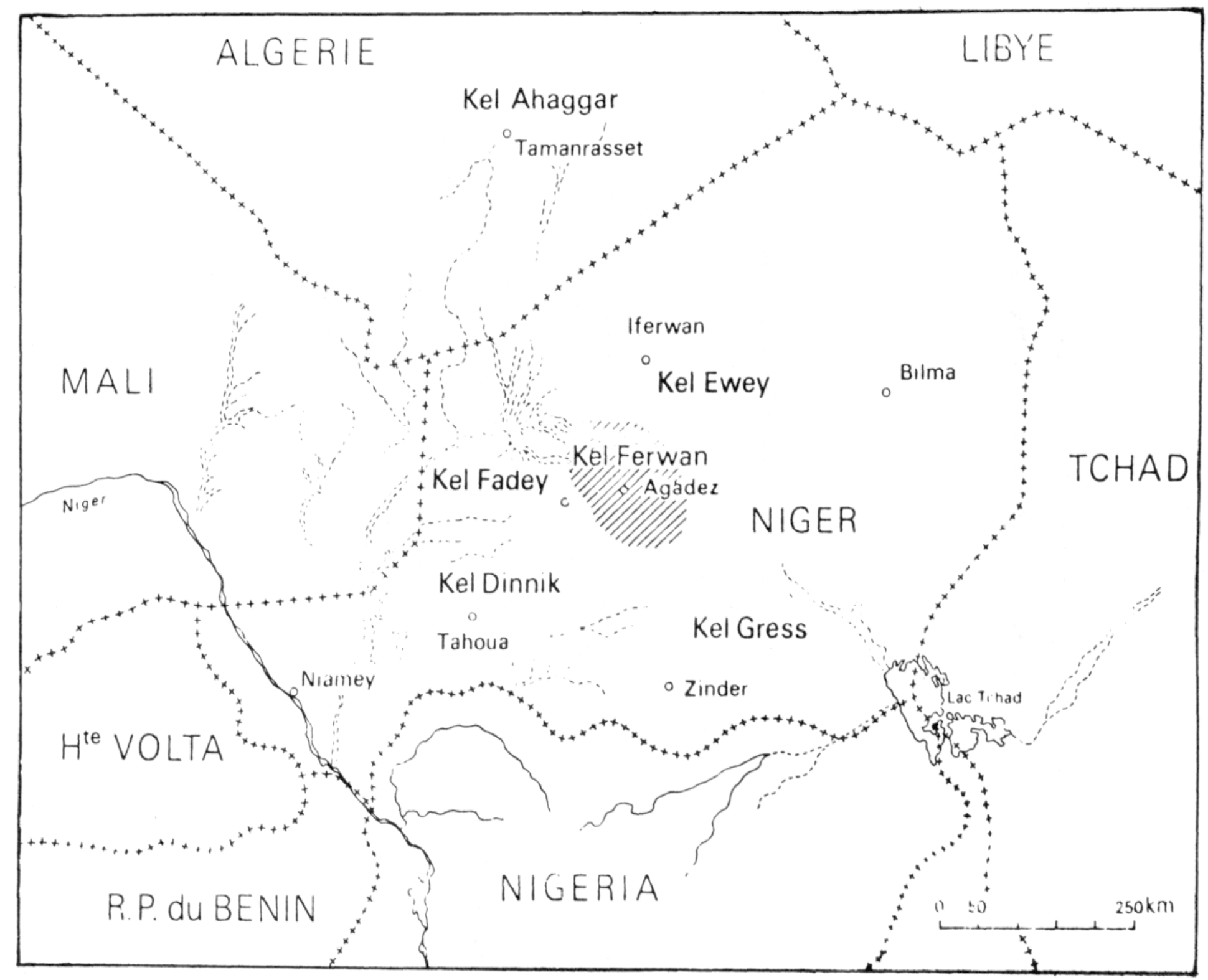

En hachure, aire de présence des Kel Ferwan dans l'Air. 


\section{LES DÉPLACEMENTS SAISONNIERS}

L'unité de résidence chez tous les Kel Ferwan est l'aghiwän (3) (pl. ighiwénän), le "campement". L'aghiwän regroupe quelques tentes, ehän (pl. inän). Il se déplace environ tous les deux mois; les déplacements sont d'abord des déplacements saisonniers.

Au mois de juin, lorsque commence la saison des pluies, temért $n$ ejenna, la "saison du nuage " appelée aussi temért en ghafäyt, "saison de l'herbe nouvelle", chaque campement s'établit dans les zones non inondables dont le sol, dur, est appelé tégdamäyt. Les précipitations sont souvent précédées d'un vent violent, l'angoya, qui emporte parfois les tentes mal arrimées. Épisodiques, elles se succèdent jusqu'en août, parfois septembre. Durant cette periode, les campements tendent à se regrouper, l'abondance des pâturages le permettant. Il n'est parfois pas besoin de tirer l'eau des puits; dans le lit des rivières, qui s’assèchent en général quelques heures après chaque précipitation, on peut trouver de l'eau à moins d'un mètre de profondeur; il suffit de creuser un puisard (qui s'effondrera au prochain orage) pour atteindre une eau filtrée par le sable. Les marigots aussi sont nombreux et on est délivré du souci d'abreuver le bétail. Si les pluies sont assez abondantes, les chèvres donnent assez de lait pour qu'il soit possible de faire des fromages qu'on consomme le plus souvent secs et pilés, mélangés au mil. Dans les régions les plus favorisées, il est également possible en cette saison de glaner des céréales sauvages ou de cueillir des baies dont on fait une sorte de confiture qu'on mélange aussi au mil (par exemple kandewe, fruit du tadänt (Boscia senegalensis).

Au mois d'octobre, les orages sont apaisés, l'herbe a déjà commencé de jaunir. C'est la saison dite de gharat, "l'herbe haute". Les campements s'établissent alors dans le lit des rivières dont le sol doux et sablonneux, l'ezizkl, est préféré à la poussière de la tégdamäyt. L'ezizkl n'est pas seulement plus confortable que la tegdamäyt; certains considèrent qu'il vaut mieux pratiquer la prière canonique de l'Islam dans l'ezizll. À tel point que les vieillards pieux vont, si le campement est installé sur la tégdamäyt, recueillir, avant de prier, un peu de sable dans le lit d'une rivière proche et le répandent devant eux avant de commencer leurs prosternations. En décembre, les vents se lèvent, les nuits deviennent froides; il faut alors déménager vers les lits des rivières les plus encaissées ou gagner l'abri de quelque bosquet. En février les vents deviennent plus chauds; commence alors la saison la plus éprouvante, ewilän. L'herbe depuis longtemps a séché, s'est faite paille. L'eau baisse dans les puits, certains même sont asséchés. C'est la saison de l'interminable attente des premières pluies, où les hommes et les troupeaux errent à la recherche de ce qu'ils peuvent trouver d'ombre et d'herbe. Les pâturages s'épuisant vite, il faut déménager plusieurs fois au cours de l'ewilän. Pour peu que les premières pluies tardent à venir, l'attente se fait inquiétude; les bètes les plus fragiles (il s'agit surtout d'ovins) meurent et chacun commence à se rappeler les années où les pluies n'étaient pas venues. Dès le premier orage, l'herbe nouvelle commence d'apparaitre, et les campements s'éloignent à nouveau des vallées. 
On voit donc l'importance de la saison des pluies, période où la nature et les hommes se régénèrent, période aussi pendant laquelle la vie est plus facile. C'est elle qui marque le début de la nouvelle année (4). Nadána, "l'an dernier ", veut dire exactement : avant la dernière saison des pluies. Même remarque pour urazän, « l'an prochain ». Awétäy, "l'année » devient au pluriel iwutiyän, terme utilisé par les Kel A haggar mais auquel les Kel Air préfèrent élan, "les feuilles ", "l'herbe nouvelle " (5). Pour savoir depuis combien d'années a eu lieu un événement, on essaie de se rémémorer combien de fois les pluies sont tombées depuis.

Le campement se déplace ainsi six ou sept fois l'an. Notons que chez les Kel Ewey à demi sédentarisés qui vivent plus au nord que les Kel Ferwan, la transhumance se réduit à un double déplacement, en début et en fin de saison des pluies. Mème réduit au minimum, le nomadisme doit tout de même comporter ce double mouvement, qui est en fait l'alternance des séjours sur la tégdamäyt et sur l'ezizél : on ne pourrait rester dans les dépressions inondables en saison des pluies, et on ne pourrait non plus demeurer sur les hauteurs, qui sont plus arides. La nomadisation des Kel Ferwan, plus ample, se construit tout de même sur ce canevas.

La succession des années, des èlän, est le retour périodique, ponctué par la saison des pluies, sur les mêmes terres de parcours. Si on évite de réinstaller un campement à l'endroit précis où on l'a installé l'année précédente, on revient tout de même à sa proximité. Un Touareg reconnaît entre mille le lieu d'un séjour antérieur. $Y$ est attachée une part de ses souvenirs, qu'il évoque à l'occasion. "Tu vois, c'est près de cet arbre que nous habitions lorsque mon frère est né "... Au bout de quelques années, quand les traces d'un ancien séjour ont totalement disparu, on peut réinstaller le campement sur son emplacement. Même si on ne reproduit pas exactement la configuration d'alors, on se souvient parfaitement de l'ancienne position des tentes et des feux, même après plusieurs années. L'écoulement du temps recouvre donc un certain jeu dans l'occupation de l'espace, et ce jeu apparaît comme le retour indéfini du même, ou tout au moins, du semblable.

Les plébéiens, qui vivent surtout de l'élevage chevrier, transhument sur des étendues assez réduites, dans un rayon d'une quinzaine de kilomètres environ. Chaque tribu a ses terres de parcours qui, de mémoire d'homme, n'ont guère varié, petit pays où chaque accident de terrain, chaque particularité topographique a un nom connu de tous, et au-delà duquel on ne s'aventure guère. Un plébéien qu'un voyage occasionnel a mis à quelques journées de marche de son campement se sent déjà en territoire étranger. Il n'aime guère s'absenter longtemps, vite atteint qu'il serait par l'esuf $n$ akal ou l'esuf $n$ aghiwän, termes qu'on peut traduire par "le mal du pays" ou " mal du campement ". Pour les vieillards, même habitant à proximité d'Agadez, venir en ville est un événement et certains n'ont pas vu Agadez depuis des années.

Les nobles, plus chameliers, évoluaient autrefois sur une zone de transhumance beaucoup plus étendue. Ils sont maintenant presque sédentarisés à Aderbissinat, à l'extrême sud du territoire des Kel Ferwan. Mais leurs serviteurs font effectuer à leurs chameaux de longs parcours. 


\section{LA TENTE}

La tente des Kel Ferwan n'est pas une tente en peau comme celle des Kel A haggar et des Ioulimeddan. Elle est faite de nattes tressées fixées par des cordages sur une armature de bois. Les nattes sont faites de palmes de palmier-doum, dont une espèce, celle utilisée, a un aspect buissonnant. Les palmes sont coupées dans le sens de la longueur et calibrées en minces rubans. La coupe de ces palmes est plutôt le travail des femmes serves. Elles les revendent sur le marché d'Agadez ou à celles des femmes libres qui ne s'adonnent pas à ce travail. La sparterie prend aux femmes une grande partie de leur temps. Les petites filles commencent très tôt à imiter leur mère, et les femmes adultes ont dans ce travail une très grande habileté.

\section{L'armature en bois de la tente}

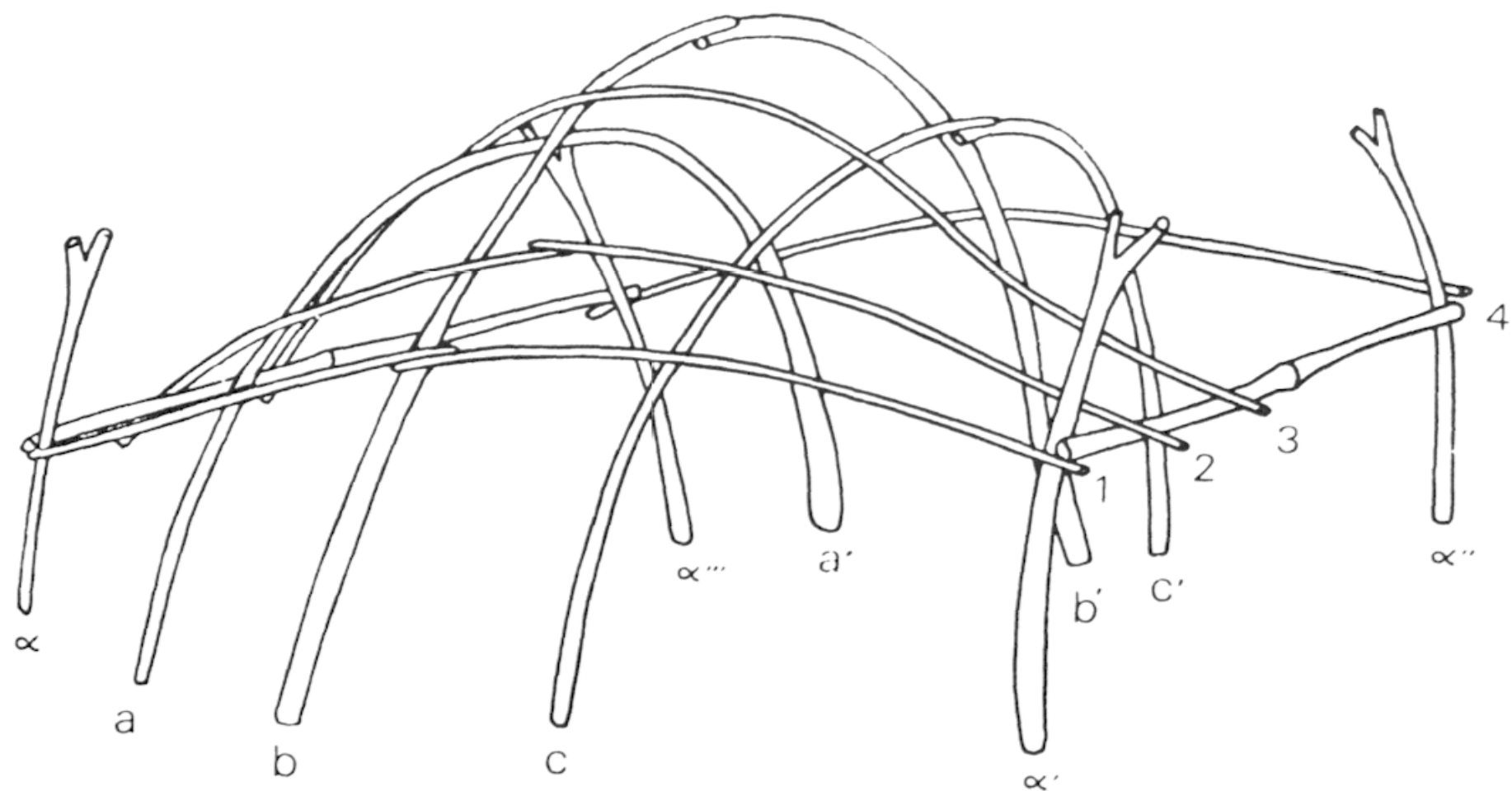




\section{L'armature vue de haut}
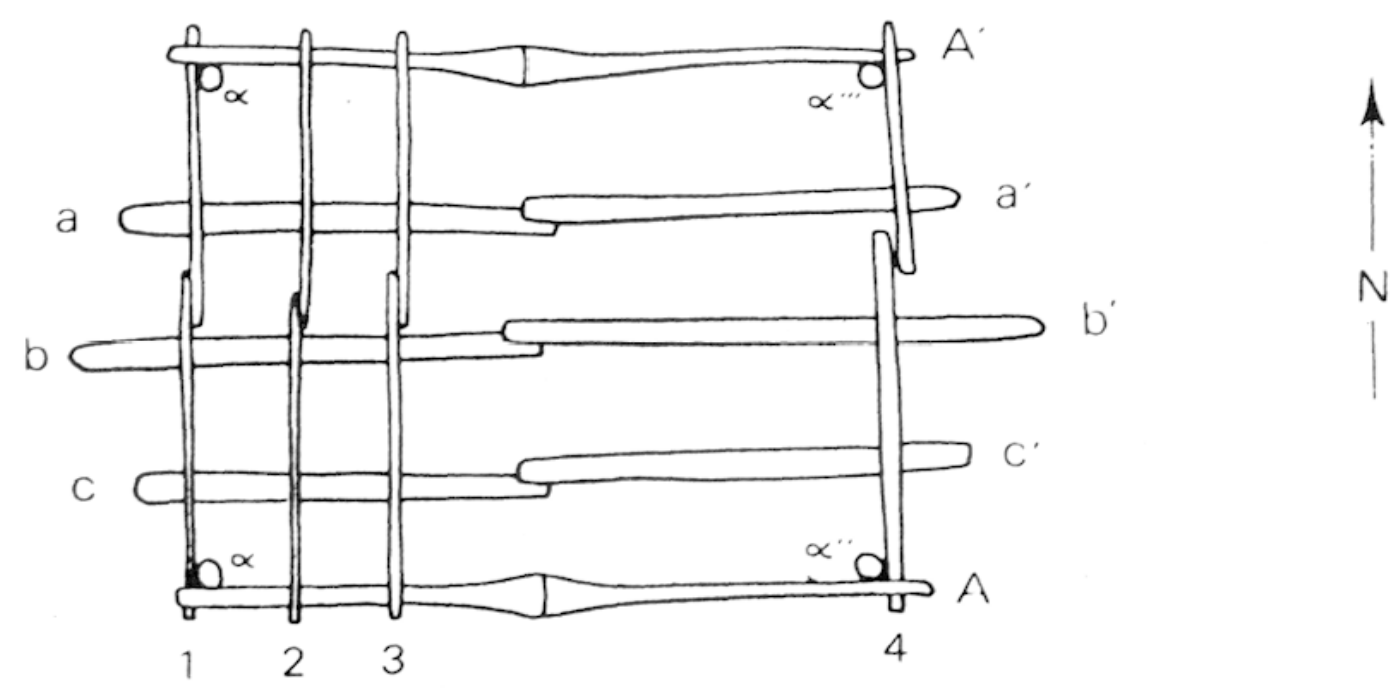

$1,2,3$, alellew (pl. ilellewän), peut se traduire par : «latte transversale ». Il y a en tout dix ilélléwän; nous n'en avons représenté que quatre. Ils sont faits avec les racines de l'afagag (Accia raddiana) ou avec des racines de termumiya, arbre soudanais que nous n'avons pu identifier.

$\mathrm{a}, \mathrm{b}, \mathrm{c}, \mathrm{a}^{\prime}, \mathrm{b}^{\prime}, \mathrm{c}^{\prime}$, terme générique : egkgu (pl. igagän), peut se traduire par " arceaux". Les igagän sont également faits avec les racines de l'afagag. Comme toutes les pièces de l'armature, les igagän sont préparés par les forgerons qui les préforment en les enterrant dans le sable après les avoir courbés.

Les arceaux a, b, c s’appellent aussi anébłttér, terme qui signifie : "celui qui permet de ne pas s'affaisser ». Les arceaux $a^{\prime}, b^{\prime}, c^{\prime}$, reposent en effet sur eux.

Les arceaux a' et c' s'appellent aussi telissawt; l'arceau c' s'appelle aussi taqkqqkwät, terme propre qui ne semble pas avoir d'autre sens. On peut remarquer que telissawt est le féminin de alissaw qui veut dire "membre de la tribu des Ilissawan". Les Ilissawan, qui vivent aujourd'hui au Damergou, comptent parmi les plus vieilles tribus du Niger; ils ont précédé les Kel Ferwan sur les terres que ces derniers occupent.

$\alpha, \alpha^{\prime}, \alpha^{\prime \prime}, \alpha "$ : tagkttewt (pl. tigettawin), peut se traduire par : « piquets ». Ils sont terminés par une sorte de fourche qui permet d'y accrocher une outre ou une calebasse de lait.

A, A', izgar, barres de support des lattes transversales.

On pose sur les lattes transversales des nattes oblongues, isalän. On peut aussi, si on est assez fortuné, poser d'abord un clayonnage de jonc appelé ewarwar. Cet ewarwar, contrairement aux nattes, n'est pas fait par les femmes du groupe mais est la spécialité d'une tribu berbère anciennement installée au Niger, les Igdalan.

Deux longues nattes rectangulaires les ighullay (sing. eghaley) font le tour de la tente. 


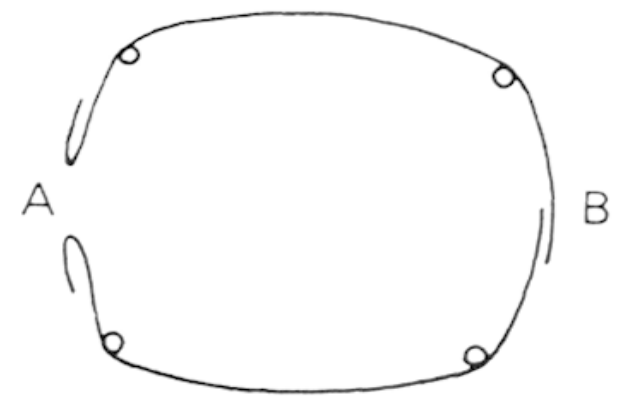

La longueur des deux ighullay est un peu supérieure au périmètre de la tente, de sorte que, s'ils sont complètement déroulés comme en $\mathrm{B}$, la tente cst totalcment fermée. On peut aussi enrouler une des extrémités comme en $A$, ménageant une ouverture. En principe, la tente est fermée du côté B, c'est-à-dire à l'est, et c'est normalement par l'ouest qu'on entre dans une tente. Dans la journée, on peut soulever un eghalley du côté où la tente est le moins exposée au soleil, pour ménager une certaine aération; à midi, la chaleur sous une tente totalement fermée serait étouffante.

Les quatre côtés de la tente ne sont pas du tout équivalents. Nous avons d'abord vu que la disposition des arceaux distingue la direction nord-sud de la direction estouest. L'informateur dit parfois que les arceaux sont comparables à la colonne vertébrale d'un individu, les lattes transversales étant semblables à ses côtes, les izgar à ses bras. L'ouverture ouest est alors comme la tête; la tente est semblable à une personne dont la tête fait face à l'ouest. Pour caractériser l'orientation de la tente, le Touareg dit qu'elle regarde vers l'ouest (ehän isaigged $i$ ataram). Il est remarquable d'ailleurs que les cases des Kel Éwey sédentarisés portent le nom de tatrémt, terme dérivé de ataram, louest (6), dont il est une sorte de diminutif.

Lorsque le soir tombe, on a grand soin de baisser l'eghalley du côté nord. C'est qu'en effet un certain danger peut venir de là. Les kel esuf, ou aljinän, les "jnoun ", êtres dont nous reparlerons, se tiennent ordinairement au nord de la tente, et c'est à la tombée du jour qu'ils sont le plus susceptibles de se manifester. De même, le lit est dans la tente orienté dans le sens est-ouest, et l'homme est au nord, la femme est au sud. L'homme ainsi barre aux "jnoun " le chemin de son épouse; ils sont moins dangereux pour lui que pour elle. En prononçant la parole bismillahu (« au nom de Dieu »), il peut les écarter, ce dont une femme est incapable. De mème, lorsqu'elle sort la nuit pour satisfaire quelque besoin, il ne faut surtout pas qu'une femme se place au nord de la tente; un homme peut à la rigueur le faire, mais il vaut tout de même mieux qu'il l'évite. Il est également incorrect d'uriner en regardant vers l'est, la direction de La Mecque. La nuit, on ne sort donc pratiquement de la tente que par le sud ou l'ouest. Des quatre côtés de la tente, le sud est le moins dangereux. Il est même source d'albaraka, de bénédiction. C'est qu'en effet, dit le Touareg, au sud se trouvent les champs de mil d'où nous viennent notre nourriture. Alors qu'au nord ne se trouvent que le désert et la faim. Certains Kel Ferwan ajoutent qu'il y a, au nord des terres qu'ils occupent, des régions frappées d'interdit, de kharam. Ce sont les régions où se trouvent les grandes mosquées de l'Aïr et où de grands saints sont inhumés. Lorsqu'il commente l'orientation de la tente, le Touareg associe donc le néfaste, les "jnoun », et l'interdit qui est aussi le sacré. 
Tout cela rappelle les anciens Arabes (Chelhod, 1965, p. 221). Le Yémen était pour eux le sud, et la racine sémitique YMN désigne aussi la droite; Shâm, la Syrie, était le nord et aussi la gauche. Le vent du nord était censé apporter avec lui la disette, par contre le Yémen est le pays florissant, l'Arabie heureuse des classiques. On pourrait invoquer un lointain emprunt si l'on oubliait qu'il y a là un schéma très général. On sait que de nombreux peuples font un rapport étroit entre la droite et l'est ou le midi, et la gauche et l'ouest ou le nord. Le nord est en général néfaste et profane et aussi féminin, et le sud, faste, sacré et aussi masculin (Hertz, 1928). Par rapport à ce schéma, les Touaregs présentent quelques différences, notons-le. Le sud est chez eux féminin, le nord tend à y être sacré et est en même temps néfaste.

L'est et l'ouest de la tente s'opposent également. C'est normalement vers l'ouest que la tente est ouverte, avons-nous dit. C'est aussi à l'ouest de la tente que l'on passe l'essentiel de la journée. On y pile le mil, on y fait la cuisine, on y mange, etc. L'est est au contraire réservé à la prière, on n'a en principe rien à y faire d'autre. Remarquons que, la "tête " de la tente étant à l'ouest, on prie en tournant le dos à la tente. L'informateur estime d'ailleurs (et c'est cohérent), que s'il existe des peuples nomadisant à l'est de La Mecque, et qui donc prient vers l'ouest, leurs tentes sont ouvertes vers l'est. Les tentes d'un campement sont volontiers alignées du nord au sud. II y a donc un côté du campement où on prie et un autre où on vaque à ses occupations. Cette règle n'est pas néanmoins aussi stricte que les règles d'orientation de la tente : un campement s'installant dans une vallée orientée dans le sens est-ouest aura parfois ses tentes parallèles à la vallée.

Le polygone formé par les points d'ancrage des piquets et des arceaux de la tente a à peu près la forme d'un cercle, c'est en tout cas ainsi que les Touaregs le voient. Ce cercle est, dit-on, analogue au cercle du monde. La terre a, en effet, selon les Touaregs, la forme d'un cercle dont la Mecque serait le centre.

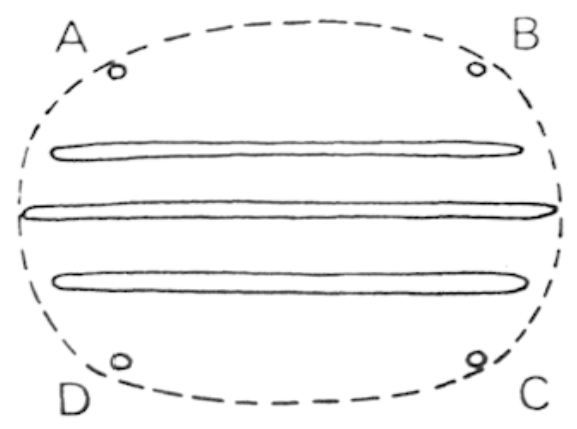

De plus les quatre piquets appelés tigkttawin, A, B, C, D sont analogues aux quatre piliers (pilier se traduit également par tagkttawt, pl. tigkttawin) qui soutiennent la voûte céleste; ces piliers sont au nord-ouest, nord-est, sud-ouest, sud-est du monde, occupant par rapport au disque terrestre la position qu'ont par rapport à la tente les quatre piquets principaux. Le toit de la tente, de forme arrondie, est analogue à la voûte céleste. Personne n'a jamais vu les quatre piliers du monde, mais, afin que les hommes croient en leur existence, Dieu a disposé dans le ciel quatrc étoiles qui en sont la réplique. Ces quatre étoiles forment un ensemble appelé tafélla, "le toit", appelé chez nous le carré de Pégase. Lorsqu'elles sont au zénith (un peu avant le lever 
du jour au mois de juillet), l'orientation du carré qu'elles forment est exactement conforme à la position des quatre piliers du monde. C'est en regardant ces étoiles que les Touaregs, disent-ils, ont appris à construire leurs tentes (7). Certains hommes ajoutent que les quatre tigkttawin (piquets de la tente ou piliers du monde) sont semblables aux quatre Piliers de l'lslam. Les informatrices n'acceptent pas cette comparaison (8).

La tente des Kel Ferwan est donc une réplique du cosmos, et est construite selon un modèle existant depuis le début des temps. Il faut remarquer que le fait est peutêtre particulier à ceux des Touaregs qui vivent dans des tentes en nattes. La tente des Kel Ahaggar est en général orientée vers le sud et son orientation n'est pas toujours stricte. La tente des Ifoghas aurait une orientation variable selon la direction du vent. Nous n'avons relevé chez les Ioulimedan, qui ont une tente en peau, aucun commentaire semblable à ce qui vient d'être présenté. Tout ce symbolisme serait donc particulier aux Kel Ferwan, Kel Ewey, Kel Fadey et sans doute Kel Gres.

\section{LES RËGLES DE RÉSIDENCE}

Le campement regroupe en principe un homme et son épouse, leurs fils, leurs brus et leurs filles non mariées, veuves ou divorcées. Mais la fréquence des divorces suppose des règles complémentaires. En cas de séparation, les enfants, se sentant plus proches de leur mère, restent toujours auprès d'elle, s'ils sont jeunes; les filles, même plus âgées, restent auprès de leur mère. Les garçons déjà grands préfèrent parfois accompagner leur père. En fait, le problème des enfants plus âgés ne se pose pas vraiment : c'est au début des mariages que les divorces sont les plus fréquents. Les jeunes gens connaissent toujours des unions malheureuses avant de s'installer dans des ménages plus durables. Un couple ayant suffisamment résisté à l'usure du temps pour que ses enfants atteignent l'adolescence ne se séparera sans doute plus. Nous n'avons eu que quatre exemples de divorces de vieux couples durant notre séjour.

Dans un de ces cas, chez des captifs, les garçons les plus âgés sont tous restés près de leur père, attribuant les torts à leur mère, alors que le père leur avait donné à choisir. Les quatre enfants les plus jeunes, deux garçons et deux filles sont restés avec leur mère. Le père s'est depuis remarié après avoir maintes fois proposé à sa femme de reprendre la vie commune.

Dans un autre de ces cas, chez des plébéiens, les enfants, toutes des fillettes, sont restées avec leur mère. Les deux fils aînés avaient depuis longtemps quitté la région, l'un étant manœuvre dans la ville minière d'Arlit, l'autre instituteur. Leur père, Zerbi, de la tribu ètrangère des Ifoghas, était installé chez les Kel Ferwan, et vivait, ce qui est exceptionnel, chez son beaupère Katia. Après son divorce, il s'est retrouvé seul, sans tente et sans campement, loin des siens, perdus de vue depuis longtemps. Après plusieurs mois de vie solitaire, il est finalement revenu dans le campement et a installé 
son lit à côté de la tente de son épouse. La chose a fait sourire, mais il n'est pas si rare que des époux âgés continuent à cohabiter tout en se considérant comme divorcés.

Même si l'informateur peut énoncer des règles, il y a toujours des cas d'espèce. La règle précédemment énoncée doit donc être corrigée ainsi : un campement regroupe un homme, son épouse, leurs fils, leurs brus, leurs filles non mariées, veuves ou divorcées et les jeunes enfants des précédents lits des femmes présentes. Cette règle supporte néanmoins des exceptions, comme nous l'avons vu. Notons qu'une veuve âgée quitte souvent le campement de ses fils adultes pour installer sa tente dans le campement de ses frères, ce qui constitue une application de la règle précédente.

La tente où réside un couple appartient à l'épouse. Une femme mariant sa fille aînée lui donne sa propre tente et s'en fait refaire une autre. Pour ses filles plus jeunes, elle s'occupe de rassembler les éléments de leurs tentes dès que le mariage a été décidé. En fait, si la mère cède la tente, elle garde pour elle une partie des éléments, ne fût-ce que le mobilier. Si elle fait faire pour sa fille une tente neuve, elle lui cède tout de même une partie des éléments de sa propre tente, de sorte qu'une mère mariant sa fille garde toujours une partie de sa tente et une fille a toujours dans sa tente quelques éléments de la tente de sa mère. Mère et fille vivent dans une tente issue de la scission d'une même tente, et pour les Touaregs il s'agit en fait de la même tente. Par rapport à ce schéma sur lequel l'informateur revient fréquemment, la différence entre fille aînée et fille cadette est finalement secondaire. Notons que deux frères vivent aussi dans des campements issus de la scission d'un même campement mais, contrairement aux campements, les tentes sont identiques, construites selon un modèle donné depuis le début des temps. Notons que chez les Kel Eway, un peu différents sur ce point, une mère commence dès leur naissance à constituer les tentes de ses filles, en en rassemblant un à un les éléments. Dans tous ces cas, qu'il s'agisse ou non de sa propre tente, unc mère donne une tente à sa fille.

\section{DU CARACTÈRE ÉPHÉMĖRE DU CAMPEMENT}

Quand meurt l'homme le plus vieux du campement, celui autour duquel le campement s'est fait, son amghar, son "chef ", c'est le fils ainé qui devient le chef du campement et c'est autour de lui que se groupent les tentes. Mais tout dépend de la personnalité de chacun. Si le fils aîné n'est pas assez fort pour s'imposer aux autres membres du campement, ils peuvent s'installer ailleurs. La mère, veuve, préfèrera peut-être s'isoler et s'occuper seule de ses chèvres, à moins, comme on l'a vu, qu'elle ne rejoigne le campement d'un de ses frères; les frères cadets enfin peuvent camper séparément en compagnie de leurs épouses et enfants. Mais nous avons vu au moins un campement se disperser avant la mort de son chef. Celui-ci, Zerni, était au dire de ses voisins un homme de peu d'autorité. En fait, la mort du campement avait peutêtre d'autres raisons. Nous avons vu des vieillards bien moins considérés que Zerni 
rester chefs de campement. Celui-ci était veuf et il n'avait donc pas de tente, la tente de son épouse ayant été, comme il se doit, repliée à sa mort et déposée sur un épineux en attendant de servir à quelque fille ou nièce. Et nous verrons plus loin qu'un homme sans tente n'a pas de poids social. Disons donc plus généralement que quand son chef meurt ou perd de son influence, le campement tend à se disperser. De toutes façons, quand le campement commence à être trop lourd, quand de trop nombreux petits-enfants entourent son chef, les fils cherchent à s'éloigner. Mais si leur père a encore assez d'ascendant sur eux, ils restent tout de même à sa proximité. Un campement meurt donc aussi bien de l'excès, du grand nombre de ses membres, que du manque, la vieillesse ou la mort de son chef. Un campement n'est donc pas un groupement stable; plus exactement sa stabilité dépend de la volonté des hommes qui le constituent; tout campement a une histoire, ses membres et ceux des campements voisins se souviennent de l'avoir vu se grouper autour d'un homme, il se dispersera un jour. C'est par le nom de son chef qu'on le désigne, on parle du "campement d'Untel ". Concrétisation de la volonté de quelques hommes de vivre ensemble, un campement peut donc cesser d'exister en tant que tel, en tant que campement d'Untel, si cette volonté fluctue ou cesse d'exister. Nous ne voulons pas dire par là que la constitution d'un campement est laissée à l'initiative individuelle, ce qui n'est pas le cas, mais que le campement est toujours provisoire, passager, comme le sont les conventions que les hommes passent entre eux. Certes, sa composition suit certaines règles et il est possible de les énoncer mais, comme nous l'avons vu, les divorces peuvent en perturber l'application et nous avons même rencontré une flagrante exception à la règle qui impose à la bru de vivre chez ses beaux-parents. C'est le cas de l'épouse de Zerbi qui vivait chez son père, Katia. Cette anomalie avait des raisons précises : Zerbi était un étranger, qui avait perdu les siens de vue. Il n'avait donc pas de campement où il pût emmener son épouse. De plus, quand sa fille s'est mariée, Katia était l'amghar, le "chef » de la tribu de Iberdiyanan. Qu'un étranger vienne s'installer chez lui était donc assez naturel : l'amghar est d'abord quelqu'un que l'on sollicite.

Le caractère, que pour l'instant nous appelons éphémère, du campement est visible dès avant sa dispersion. Pour le sentir, poussons plus avant la description que nous en faisons. Le petit bétail est installé pour la nuit dans des enclos circulaires (afarag, pl. ifergän) faits de branches d'épineux. La règle de répartition des enclos n'est pas fixe. Dans certains campements, il n'y a que deux enclos, un pour les ovins, un pour les caprins, voire même un seul enclos si le bétail est peu nombreux. Dans d'autres, il y a un enclos par couple, donc par tente. En fait, il semble que si le couple est jeune, si le fils craint encore son père, il met alors son bétail dans l'enclos de son père. Il n'y a alors dans le campement qu'un enclos, ou bien un enćlos pour les ovins et un pour les caprins. Par contre si le père est âgé, ne peut plus guère travailler, ou simplement commence à perdre de son importance dans le campement, chaque tente a son enclos. L'existence de un ou plusieurs enclos dépend donc de la cohésion du campement derrière son chef. Un campement où l'ancien prend seul les décisions n'a qu'un enclos, un campement où les fils ont assez de dynamisme personnel peut en avoir plusieurs. Le nombre d'enclos, dans ses variations possibles, est donc l'illustration du devenir du campement. Celui-ci commence par n'avoir qu'un enclos, puis au 
moins après la mort de son chef en a plusieurs; c'est le début de la dispersion future. La non-fixité des règles de répartition des enclos est donc l'illustration du caractère dynamique du campement.

Les enclos sont construits par les hommes. Nous verrons que quand un campement déménage, les hommes choisissent d'abord le nouvel emplacement et $y$ installent le ou les enclos. Les femmes viennent ensuite installer leurs tentes sur le site qu'on leur a délimité. Les campements s'installent donc autour d'enclos construits par des hommes et leurs habitants se regroupent autour d'hommes, un père et ses fils.

\section{LE DESTIN DES HOMMES ET DES FEMMES}

Nous avons senti ce que pouvait avoir de fragile ce regroupement d'hommes vivant dans des tentes de femmes. Nous allons préciser cela. La façon même dont nous parlons ici de l'habitat suggère un statut différent des hommes et des femmes par rapport à lui.

Un enfant, garçon ou fille, vit d'abord dans la tente de sa mère, très près d'elle. Les enfants sont allaités jusqu'à trois ans par leur mère, qu'ils appellent parfois par son prénom et qui ne leur refuse rien. Le nom qu'elle leur donne en retour, en général un tendre diminutif, n'est pas celui qu'utilise leur père (9). Des enfants déjà grands sucent encore le sein maternel, pourtant tari depuis longtemps. Mais quand un garçon atteint l'adolescence, quand s'éveille sa virilité, la cohabitation avec le compagnon de sa mère (qui peut ne pas être son père) devient pour lui délicate. Encore que j'ai vu des jeunes gens de près de vingt ans vivre sous la tente maternelle et ne pas pouvoir s'en absenter longtemps sans qu'il leur en coûte. Mais en général l'adolescent commence à déserter la tente de sa mère, préférant se constituer un précaire abri à l'aide de branchages et de quelques nattes, abri qu'il partagera peut-être avec un compagnon d'âge. Il peut aussi chercher l'hospitalité de quelque cousine momentanément sans époux. C'est aussi à ce moment de sa vie qu'il commence à porter le voile facial. L'adolescent devra attendre de trouver une épouse pour pouvoir à nouveau vivre dans une tente. Il peut attendre de nombreuses années, un homme restant souvent célibataire jusqu'à trente ans. Se marier se dit pour un homme ägu-ehän : "faire une tente ". On dit d'un homme qu'il " pénètre dans la tente de sa femme " au moment où il l'épouse (iggkz ehän en témtut net). Il devient alors le " maître d'une tente " (messhish $n$ ahän), du moins tant qu'il ne divorce pas. Car dans ce cas, il se retrouve dans la position d'un adolescent et son poids social en souffre, fût-il un vieillard. Un veuf non plus n'a pas de tente et nous avons vu qu'il supporte les mêmes inconvénients. S'il a une fille momentanément sans époux, elle se fera un devoir de l'héberger, mais s'il n'a que des fils, il doit s'attendre à un veuvage amer.

La fillette connaît elle aussi une période de grande intimité avec sa mère, mais les incertitudes de l'adolescence masculine lui sont épargnées; la jeune fille est mariée très tôt, dès sa nubilité et parfois même avant. C'est assez souvent son cousin croisé patrilatéral qui l'épouse, mariage en général assez éphémère, mais que lui importe; elle a maintenant une tente, et pour toujours. Un homme peut parfois en être le maître, 
mais une fois qu'elle a obtenu une tente, une femme ne cesse jamais d'en avoir. Dans la langue poétique ou les plaisanteries grivoises, une femme est parfois appelée une tente. Dans la langue de Tahoua et cette fois dans la conversation courante, l'épouse d'un homme est normalement appelée "sa tente ". Notons chez les Kel Ahaggar l'emploi du terme poétique tin hanän, "celles des tentes", qui désigne un peu cavalièrement "les femmes du campement " (Père de Foucauld 1952, t. II, p. 609). L'expression ne semble pas utilisée par les Kel Ferwan. Notons que chez ces derniers, l'utérus d'une femme est volontiers désigné par le terme ehän, "tente ". On peut dire aussi ehän én barar, la tente de l'enfant.

Une pratique montre d'ailleurs à quel point la femme est plus que l'homme, proche de la tente : il est dangereux de poser un petit garçon ne marchant pas encore sur le bord d'une tente. Si par mégarde on s'oublie à le faire, il faut immédiatement le poser ainsi successivement sur sept autres tentes. Si on s'abstenait de le faire, il pourrait tomber malade et peut-être mourir. Par contre, une fillette ne risque rien à être ainsi posée sur une tente, car, dit l'informateur, la tente lui appartient et elle ne risque rien à être mise ainsi en contact avec elle (10).

Lorsqu'au soir de ses noces la jeune mariée est conduite par ses parentes vers la tente qui sera la sienne, et où l'attend le mariè, les femmes qui l'accompagnent lui chantent ce chant :

« Ô ma fille, ô ma fille, ô ma fille, jeunes filles

Ô ma fille, ô ma fille, ô ma fille, vous qui m'êtes semblables

Ô ma fille, ô ma fille, la nuit dernière, profond était mon sommeil

Ô ma fille, ô ma fille, lorsque Khamatan s'est penchée vers moi

me disant : " ta fille prend époux ",

Ô ma fille, ô ma fille, je me suis éveillée pleine de joie,

Ô ma fille, ô ma fille, j’ai saisi mon voile en hâte

Ô ma fille, ô ma fille, je me suis mise à courir çà et là dans la campagne

Ô ma fille, ô ma fille, j’ai coupé les branches devant devenir les arceaux de la tente.

Ô ma fille, ô ma fille, j'ai fait construire la tente vaste qui sera la tente des épousailles

Ô ma fille, ô ma fille, la ruelle du lit y est spacieuse

Ô ma fille, ô ma fille, nous y plaçons un lit fait de nervures de feuilles de palmier

Ô ma fille, ô ma fille, un lit digne d'une femme d'une grande beauté

Ô ma fille, ô ma fille, une femme dont les incisives sont un peu espacées

Ô ma fille, ô ma fille, Dieu soit remercié pour son ventre bien rond

Ô ma fille, ô ma fille, qui descend jusqu'à ses genoux

Ô ma fille, ô ma fille, ses fesses sont comme des écuelles

Ô ma fille, ô ma fille, ses cuisses comme des auges

Ô ma fille, ô ma fille, son vagin est là entre ses cuisses ». 
Chaque vers, chanté par une soliste,est repris par ses compagnes. La soliste fait ici parler la mère de la mariée. Elle s'adresse aux jeunes filles, ses "semblables ». La tente est exclusivement affaire de femmes. Le chant emploie des termes voisins pour décrire la tente et celle qui l'habitera. Après avoir évoqué la beauté de la tente, vaste et spacieuse, elle prête également à la mariée de vastes proportions. Les termes de la comparaison sont même des ustensiles de cuisine, appartenant au mobilier de la tente. Il y a dans ce chant quelque chose de cette identification de la femme à la tente que nous avons évoquée plus haut.

D'ailleurs, même n'ayant pas encore de tente, une jeune fille ne connaît pas les errances de ses frères. Elle peut sans trop de problèmes cohabiter avec ses parents ou ètre l'hôtesse de quelque soeur aînée momentanément célibataire. Chez les nobles et les plébéiens, les femmes divorcées ont une situation assez confortable. Elles vivent dans le campement de leurs parents, maitresses de leur tente, disposant des troupeaux dont elles ont été dotées au moment de leur mariage. Leur tente, à la nuit tombante, est parfois le lieu de rendez-vous des jeunes gens et jeunes filles venus à un ténde, réunion où parfois l'on chante et danse et où le plus souvent on se contente de deviser galamment. C'est le seul cas où une tente a un certain rôle en tant que telle, indépendamment du campement dont elle fait partie.

\section{LE DÉMÉNAGEMENT}

Cette différence entre hommes et femmes dans leur manière d'habiter tentes et campements devient palpable quand le campement se déplace.

Lorsque les pâturages alentour commencent à s'épuiser nous avons vu que les hommes partent à la recherche d'autres pâturages et choisissent un autre emplacement, amkjłr, où ils installent les enclos à petit bétail. Quand l'aire du nouveau campement a été délimitée par les hommes, les femmes démontent leurs tentes, ce qu'elles sont les seules à savoir faire. Les tentes repliées sont chargées sur les ânes ou les chameaux et la population du campement se met en branle, poussant son bétail devant elle, chèvres surtout chez les plébéiens, chameaux plutôt chez les nobles. Les femmes remontent leurs tentes sur le site qu'on leur a choisi, en commençant à disposer ce qui se tient à l'intérieur de la tente, c'est-à-dire essentiellement le lit et divers piquets ouvrés, sur lesquels on installe la lingerie d'une part et les provisions d'autre part. Si les habitants du campement arrivent sur le nouveau site trop tard pour que les tentes soient montées avant la tombée de la nuit, ils dorment en plein air, mais dans leur mobilier déjà en place : une tente est autant un mobilier qu'un abri de natte.

Les femmes s'enduisent d'une poudre ocre, la tamazgut, issue du broyage d'une terre qu'on trouve du côté de Tafadek, un peu au nord d'Agadez. La tamazgut a la réputation de protéger du soleil. En fait, les femmes sont toujours exposées au soleil, quand elles vont puiser de l'eau ou faire paitre des chèvres et le déménagement est le seul moment où elles utilisent ce fard. Mais c'est aussi le seul moment où elles sont sans tente. Leurs tentes étant repliées, elles semblent plus vulnérables au soleil. Certes 
les hommes sont voilés mais les adolescents ne le sont pas et ils ne s'enduisent pas le visage de tamazgut alors que les fillettes le font. Nous comptons montrer dans une publication ultérieure à quel point le voile est bien plus qu'un simple vêtement et est lié à la position des hommes hors de la tente. Tout se passe comme si les femmes ont plus que les hommes à craindre l'absence de tente. Remarquons que chez les Kel Ahaggar, Duveyrier signale qu'on utilise une poudre ocre comme "cosmétique tinctorial et hygiénique de la peau en vue de la préserver des influences atmosphériques extérieures " (Duveyrier, 1864, p. 146). Cette poudre s'appellerait tamagóhit, terme qu'on peut avec vraisemblance considérer comme parent de tamazgut. Lhote note également que chez les Kel Ahaggar, les femmes allant visiter une de leurs parentes venant d'accoucher se mettent de l'ocre jaune autour des yeux et de la bouche pour éloigner les "mauvais esprits" qui rôdent autour de la tente de l'accouchée. Ces mauvais esprits dont parle Lhote correspondent aux "jnoun " que nous avons déjà rencontrés. (Lhote, 1955, p. 325). Les Kel Ferwan ne nous ont pas parlé de la tamazgut comme un remède des "jnoun", mais ils s'en servent aussi comme remède des oreillons (igerzän); or cette maladie est parfois attribuée à la togérshit, la jettature, phénomène analogue à l'action des "jnoun ». Retenons donc que lorsque leurs tentes sont repliées les femmes sont exposées à un danger peut-être plus grand que celui que peut faire courir la simple exposition au soleil, et qui semble avoir quelque chose à voir avec les «jnoun». Ces «jnoun" qui se tiennent à l'extérieur de la tente et que les hommes tiennent normalement à distance semblent plus menaçants pour les femmes maintenant que la tente est démontée. Notons que hez les Kel Ahaggar (Foley, 1930, p. 12) le vélum des tentes est teinté à l'ocre rouge. Les femmes Kel Ferwan, en mème temps qu'elles se préservent des "jnoun ", semblent s'identifier à leurs tentes et les «jnoun " qui d'habitude assiègent les tentes tourmentent maintenant les femmes elles-mêmes.

Ce fard mérite un autre commentaire : en deux autres occasions, les femmes se fardent d'une manière qui rappelle la tamazgut. Avant les fêtes et en particulier les mariages, elles se teignent le visage avec du henné. La peau prend alors une teinte argileuse qui n'est pas sans rappeler la couleur que lui donne la tamazgut. Il y a, sur ce point, une analogie entre le mariage et le déménagement, qui tient à une analogie plus profonde : le mariage et le déménagement sont les deux seules occasions où des tentes se déplacent. La tamazgut est bien liée au déplacement des tentes et non pas seulement au soleil. Notons enfin que lors de la fête dite de Ganni, qui se déroule annuellement au mois de Safar, les femmes de certaines tribus Ifoghas et Ikazkazan s'enduisent le visage d'une teinture végétale jaune provenant du pays haousa, la taserwaq. Or, la tasérwaq est aussi utilisée pour teindre les piquets d'une tente neuve. Une femme est donc ici parée comme peut l'être une tente et la tamazgut se trouve être un lointain rappel de cette parure. L'identité de la femme et de la tente est soulignée au moment du Ganni et suggérée lors du déménagement. D’autre part, au moment du Ganni, est mimé le rapt d'une femme. Une certaine analogie existe chez les Touaregs entre le mariage et le rapt d'une femme. Nous voyons donc ici affirmée, avec une intensité d'ailleurs variable, la série d'identités: déménagement $=$ déplacement d'une tente (non exempt de dangers) $=$ rapt $=$ mariage . 


\section{LA TENTE ET LE CAMPEMENT COMME VALEURS}

Il est sans doute perceptible, surtout après ce que nous avons dit du déménagement, qu'il y a plus dans le jeu des tentes et des campements qu'une certaine forme d'aménagement de la transhumance. Et c'est sur ce point que nous allons maintenant insister.

Un campement est à la fois un simple groupement de tentes et l'expression d'un certain jeu social. En cela, le mot aghiwän se distingue de amłzzagh, qui ne désigne que le groupement de tentes. De plus, une tribu est un ensemble de campements et non un ensemble de tentes. Une tente " ne se voit pas " de l'extérieur du campement dont elle fait partie, elle n'a pas d'existence en dehors de lui. C'est seulement à l'intérieur de ce campement qu'elle a une certaine individualité, que ses occupants forment une entité distincte. Aux yeux d'un Touareg d'un autre campement, un homme appartient au campement d'Untel et non à la tente d'Une Telle. Expression d'une certaine relation entre ceux qui le composent, le campement est de surcroit ce qui les met en relation avec les autres campements, avec le reste de la tribu. La tente, quant à elle, nous est apparue comme étant du domaine des femmes, mais nous avons vu aussi que sans tente, l'homme n'a aucun statut social et n'a donc pas part au jeu des campements. Et nous avons vu que le veuvage ou le divorce, donc la privation de tente, peut replonger l'homme dans l'inexistence sociale des adolescents.

Le campement se construit autour d'hommes, mais ceux-ci ne sont rien sans des tentes et inversement une tente n'est pas concevable ailleurs que dans un campement : quand une veuve décide de vivre à l'écart du campement de ses fils et qu'elle ne réintègre pas celui de ses frères, elle replie parfois sa tente, n'en gardant que le minimum d'éléments. De plus pour qu'une femme acquière une tente, doit se jouer cette partie essentielle de la relation entre campements qu'est le mariage.

Nous avons vu que la bru vit en principe chez ses beaux-parents. Le mariage est donc le départ d'une femme (d'une tente) dans un autre campement. Le campement est donc constitué par le mariage, mouvement incessant qui, en même temps, le met en question, migration des tentes à travers les campements. Il est lié aux hommes, dont nous savons aussi qu'ils affrontent l'extérieur avant les femmes au moment des déménagements. Le campement est donc, de ces deux manières, tourné vers l'extérieur et s'oppose ainsi à la tente, liée aux femmes, et que, lors des déménagements, celles-ci édifient en commençant par l'intérieur.

Cette opposition de la tente et du campement, dite ici en termes spaciaux, peut aussi se dire en termes temporels. Nous avons parlé du caractère "éphémère " du campement. Ceci n'est pas quelque accident de sa nature. Il faut que le campement soit éphémère, dynamique en fait pour pouvoir être. A la tente, réplique du cosmos, et à ce titre image d'une certaine permanence, il oppose la succession ininterrompue des étapes du jeu social; on peut aller plus loin et dire que le campement est l'instance où se déploie la temporalité. Et là est le véritable sens de ce que nous avons appelé jusqu'ici son caractère éphémère. Rappelons-nous en effet que dans les emplacements 
successifs des campements, on lit que les années passent. Les campements reviennent toujours aux mêmes emplacements, mais ceux qui se souviennent mesurent à l'occasion de chaque retour combien les choses ont changé depuis la dernière fois. De plus, la répartition des enclos autour desquels s'établit le campement est le signe même que le campement est à tel ou tel moment de sa vie. Dans le campement s'inscrivent donc - et dans le même mouvement - ce qu'on pourrait appeler le temps cosmique et le temps social, c'est-à-dire la succession des saisons et celle des événements sociaux. A la succession des saisons est liè le déplacement des campements, qui est d'abord celui des enclos. A la succession des événements sociaux est liée la modification des campements, visible d'abord dans la modification du statut des enclos. Les moments forts de ces deux successions sont le mariage et le déménagement, dont, nous l'avons vu, l'analogie est précisément soulignée. En tout cela, là encore, nous pouvons dire que le campement est à la tente ce que la temporalité est à la permanence.

\section{OUVRAgES CITÉS}

Chelhod (Joseph), Les structures du sacré chez les Arabes, Paris, Maisonneuve et Larose, 1964,288 p.

Duveyrier (Henri), Les Touareg du nord. Paris. Challamel l'ainé, 1864, 499 p.

Foley (Dr Jean), Mceurs et médecine des Touaregs de l'Ahaggar. Alger. Archives de l'Institut Pasteur. t. 8. pp. 167-287.

Foucauld (Père de), Diclionnaire touareg-français, Paris, Imprimerie Nationale, 4 tomes, 1952.

Hertz (Robert), "Sur la prééminence de la main droite", in Sociologie religieuse et folklore. PUF. 1980. $208 \mathrm{p}$.

Khawad (Makhmoud), “La tagdudt ", Tisuraf no 3, Universite Paris VIII, 1979, pp. 79-82.

Lhote (Henri), Les Touaregs du Hoggar, Paris, Payot, 1955, 467 p.

\section{NOTES}

(1) Une partie du matériel exposé dans cet article a été utilisé dans notre thèse de troisième cycle. $L a$ tente et l'esuf, Université de Paris VII, novembre 1979, 485 p. ronéo.

(2) Nous traduisons ainsi le mot amajłgh qui désigne en principe les Touaregs de l'aristocratie. Amghid sera traduit par plébéien. En utilisant cette terminologie, qui s'autorise d'ouvrages antérieurs, nous ne visons à rien d’autre quà la commodité et ne prètendons pas rendre compte du contenu des catégories que les Touaregs rendent par ces mots.

(3) Le mot amezzagh est également employé mais il est plus rare.

(4) Il semble que le dèbut de la nouvelle année était autrefois situé à l'apparition d'une étoile, ghadat, visible à partir de la fin août (voir Makhmud Khawad, 1979.pp. 79-82). Nous n'avons pas pu identifier cette étoile, vraisemblablement située à l'ouest de Sirius.

(5) A wétäy peut désigner aussi bien l'année solaire que l’année musulmane : elän désigne exclusivement des annèes solaires.

(6) Alaram signifie d'abord: "le bas, le contrebas, l'aval ". C'est en effet vers l'ouest que le soleil "descend » et à louest qu'il " tombe ". Le mot \&nndg qui correspond à " est " signifie de mème à l'origine : " le haut, l'aval ".

(7) Il y a là une certaine parenté avec un fait arabe : des traditions considèrent que la Ka'ba est une tente du ciel, envoyé par Dieu à Adam (Chelhod, op. cit.. 221).

(8) Les Piliers de IIslam sont en fait cinq et représentent les devoirs fondamentaux du croyant. La remarque est due à des informateurs peu ou pas lettrès. Elle est désapprouvèe et par les femmes et par les lettrès. du moins les plus èrudits. 
(9) Un Touareg a donc deux prénoms, un que lui donne sa mère et un autre que lui donne son père.

(10) C'est ce dernier commentaire de l'informateur qui nous paraît ici intéressant. Mais cette pratique reste globalement assez mystérieuse : c'est par des contacts répétés avec la tente que l'on écarte le danger qu'a fait courir un premier contact avec elle. Ce qui d'ailleurs intéresse l'informateur ce n'est pas le fait tout entier, qu'il prend comme tel sans s'interroger sur lui, mais la différence de traitement qu'y reçoivent filles et garçons.

\title{
Résumé
}

Nous livrons ici une série de faits ethnographiques recueillis chez les Touaregs Kel Ferwan (nord du Niger) et restés jusqu'ici un peu inaperçus. La tentc des Kel Fcrwan cst considérée comme le domaine de la femme. Tout d'abord la tente où vit un couple appartient à l'épouse. Les tentes se transmettent de mère en fille. Ensuite, une femme tend à être identifièe à sa tente. Par ailleurs, la tente est considérée comme une représentation du cosmos et elle est construite selon un modèle immuable depuis, dit-on, le début des temps. Les tentes sont regroupées en campements, qui constituent des unités de résidence patrilocale. Le campement est le domaine des hommes. Au niveau du campement, la société s'inscrit dans l'histoire et la temporalité. La tente et le campement s'opposent donc comme domaine des femmes et domaine des hommes et aussi comme lieu de l'immuable et du temporel.

\begin{abstract}
Here are a series of ethnographical facts collected from the Kel Ferwan Touaregs (in the north of Niger) and which up to now have been granted little attention. For the Kel Ferwan the tent is considered as pertaining to the women's domain. First of all the tent where a couple lives belongs to the wife. Moreover it is handed down from mother to daughter, and then a woman tends to be identified with her tent. The tent is considered as symbolizing the cosmos, and it is said to be built according to a pattern unaltered from beginning of times. The tents are gathered to form encampments, which constitute patrilocal units. The encampment is the domain of the men, the place where society pertains to history and time. So that the tent and the encampement are opposite, one being the domain of the women and of immutability, the other that of the men and of history.
\end{abstract}

